\title{
Guest Editors' Introduction for Special Issue on Service Management for the Internet of Things
}

\author{
SAMIR TATA, IBM Research, USA \\ QUAN Z. SHENG, Macquarie University \\ ELENI STROULIA, University of Alberta
}

\begin{abstract}
ACM Reference format:
Samir Tata, Quan Z. Sheng, and Eleni Stroulia. 2018. Guest Editors' Introduction for Special Issue on Service Management for the Internet of Things. ACM Trans. Internet Technol. 19, 1, Article 6 (December 2018), 3 pages. https://doi.org/10.1145/3293539
\end{abstract}

\section{INTRODUCTION}

The Service-Oriented Computing (SOC) paradigm has evolved over the years to be a comprehensive, interdisciplinary methodology for modern software development. It has gone beyond just a "software componentization technology" to embody and express the software manifestation of a trend that transforms our society from an industrial, production-centric economy into a digital, service-centric economy. Indeed, SOC has already emerged as the principle methodology for software engineering of large complex web-based systems, including cloud- and grid-based systems (Bouguettaya et al. 2013).

Nowadays, the SOC paradigm is, however, facing new challenges imposed by the Internet of Things (IoT) that has received significant attention both in industry and in the academic community. IoT enables us to turn a tangible entity into a node on the Internet. It is estimated that more than 50 billion Internet-connected things will be in use worldwide by 2020 . Consequently, theoretical foundations and engineering methodologies and techniques for SOC that have been developed for "traditional" systems and environments are more and more questioned when it comes to their application to the IoT systems (Bouguettaya et al. 2017; Qin et al. 2016). IoT poses several fundamental challenges on service-management tasks. Resource constraints are the first concern for most IoT devices and traditional SOC standards (e.g., SOAP and BPEL) are too heavyweight to be applicable in IoT. In addition, unlike the traditional service settings, in the Internet of Things environment, functionality of system components is typically heterogeneous, multi-layered (e.g., IoT devices, data, services), dynamic, and context-aware, bringing significant complexity for service management in IoT.

This special issue focuses on techniques for establishing and maintaining IoT infrastructures, platforms and applications based on the SOC paradigm, addressing questions around the design,

\footnotetext{
Authors' addresses: S. Tata, IBM Research, USA; email: samirtata@gmail.com; Q. Z. Sheng, Department of Computing, Macquarie University, Sydney, NSW 2109, Australia; email: michael.sheng@mq.edu.au; E. Stroulia, Department of Computing Science, University of Alberta, Edmonton, Alberta, T6G 2E9, Canada; email: stroulia@ualberta.ca.

Permission to make digital or hard copies of part or all of this work for personal or classroom use is granted without fee provided that copies are not made or distributed for profit or commercial advantage and that copies bear this notice and the full citation on the first page. Copyrights for third-party components of this work must be honored. For all other uses, contact the Owner/Author.

(C) 2018 Copyright held by the owner/author(s).

1533-5399/2018/12-ART6

https://doi.org/10.1145/3293539
} 
deployment, configuration, and control of services-based IoT systems and applications. Topics of interest include design-time and run-time management of IoT services and service-based IoT systems and applications.

\section{IN THIS SPECIAL ISSUE}

This special issue aims at presenting the latest developments, trends, and solutions of service management for the Internet of Things. There were 20 submissions and four articles were selected after several rounds of rigorous review by the guest editors and invited reviewers.

The first article, by Taherkordi, Eliassen, McDonald, and Horn, "Context-Driven and Real-Time Provisioning of Data-Centric IoT Services in the Cloud," proposes a software framework for realtime provisioning of cloud-based IoT services and data, driven by their contextual properties. The proposed approach structures the description of data-centric IoT services and their real-time and historical data in a hierarchical form, in accordance with the context model of the end-user applications. Thanks to this approach, end-user applications can access IoT services and subscribe to their real-time and historical data at different contextual levels, e.g., from a municipal district to a single street, in smart-city use cases.

The second article, by Mezghani, Exposito, and Drira, "An Autonomic Cognitive Pattern for Smart IoT-based System Manageability: Application to Comorbidity Management," proposes a set of design patterns that reduce the system-design complexity, by selecting the appropriate combination of patterns based on the system requirements. The authors use an autonomic cognitivemanagement pattern to develop processes that detect and manage context changes. These processes are coordinated based on cognitive mechanisms that enable the system to perceive and understand the meaning of the received data for decision making, as well as discovering new processes that meet the requirements evolution at runtime.

The third article, by Mahmud, Ramamohanarao, and Buyya, "Latency-aware Application Module Management for Fog Computing Environments," proposes a latency-aware applicationmodule management policy for the fog environment. The authors demonstrate that the proposed policy meets the diverse service-delivery latency and amount-of-data signals to be processed in per time-unit for different applications. The policy aims to ensure that the application meets the Quality of Service requirements by satisfying service-delivery deadlines and optimizing resource usage in fog environments.

The fourth article, by Konstantinidis, Irakleous, Georgiou, Zeinalipour-Yazti, and Chrysanthis, "IoT Data Prefetching in Indoor Navigation SOAs," studies the problem of prefetching IoT data from an Internet-based indoor navigation SOA to a mobile device, without knowing the user's destination during navigation. The proposed approach analyzes building topology in an offline phase to identify important areas inside building complexes, such as malls, hotels, and campuses. The identified areas become virtual targets in an online phase to search for important and likely to be visited areas, that have to be prefetched.

The articles included in this special issue cover several important topics and present some of the key directions in this vibrant and rapidly expanding area of research and development. We hope that the selected articles provide the community with a better understanding of the current directions and areas of focus in the future and that they inspire your own work.

\section{ACKNOWLEDGMENTS}

We thank all the authors for considering this special issue as an outlet to publish their research results in the area of service management for the Internet of Things. We also thank the referees who provided very useful and thoughtful feedback to the authors. Finally, we express our gratitude to 
the Editor-in-Chief, Professor Munindar P. Singh, for his kind support, advice, and encouragement throughout the preparation of this special issue.

\section{REFERENCES}

Athman Bouguettaya, Quan Z. Sheng, and Florian Daniel. 2013. Advanced Web Services. Springer.

Athman Bouguettaya, Munindar Singh, Michael Huhns, Quan Z. Sheng, Hai Dong, Qi Yu, Azadeh Ghari Neiat, Sajib Mistry, Boualem Benatallah, Brahim Medjahed, Mourad Ouzzani, Fabio Casati, Xumin Liu, Hongbing Wang, Dimitrios Georgakopoulos, Liang Chen, Surya Nepal, Zaki Malik, Abdelkarim Erradi, Yan Wang, Brian Blake, Schahram Dustdar, Frank Leymann, and Michael Papazoglou. 2017. A service computing manifesto: The next 10 years. Commun. ACM 60, 4 (2017), 64-72.

Yongrui Qin, Quan Z. Sheng, Nickolas J. G. Falkner, Schahram Dustdar, Hua Wang, and Athanasios V. Vasilakos. 2016. When things matter: A survey on data-centric internet of things. F. Netw. Comput. Appl. 64 (2016), 137-153. 